\title{
Huge Intravaginal Pedunculated Fibroid Embolization and Resectoscopy-A Case Report and Review of Literature
}

\author{
Hirides Petros*, Karygiannis Michalis, Tsoumbis Athanasios, Hirides Savvas \\ Athens Medical Center, Marousi, Greece \\ Email: *dr.hiridis@gmail.com
}

How to cite this paper: Petros, H., Michalis, K., Athanasios, T. and Savvas, H. (2019) Huge Intravaginal Pedunculated Fibroid Embolization and Resectoscopy-A Case Report and Review of Literature. Surgical Science, 10, 222-228.

https://doi.org/10.4236/ss.2019.107024

Received: June 11, 2019

Accepted: July 16, 2019

Published: July 19, 2019

Copyright (c) 2019 by author(s) and Scientific Research Publishing Inc. This work is licensed under the Creative Commons Attribution International License (CC BY 4.0).

http://creativecommons.org/licenses/by/4.0/

\begin{abstract}
Background: Submucosal fibroids account for approximately 15 - 20 percent of total fibroids. Rarely, they prolapse. Common size is $2-6 \mathrm{~cm}$, because larger fibroids are unlikely to fit through the cervix. Larger fibroids are associated with significant bleeding and pose a surgical challenge. Case Presentation: We present a 38-year-old woman nulliparous with an intravaginal pedunculated fibroid, $12 \mathrm{~cm}$ in diameter. She presented with metrorrhagia and an intense malodorous vaginal discharge, irresponsive to oral therapy. The patient had a history of resectoscopic fibroid enucleation, 7 months earlier, followed by insertion of an (intra-uterine device) IUD. There was no uterine prolapse. Management involved bilateral uterine artery embolization, followed by hysteroscopic excision with rigid resectoscope. The fibroid was "delivered" transvaginally intact. The uterus was preserved. The IUD was partly buried within the mass. Blood loss was negligible. Patient recovery was quick and uneventful. At 6-months follow up, pelvic anatomy has been restored. Conclusion: Large pedunculated fibroids are very rare. Embolization of uterine arteries has proven a valuable tool in challenging gynecologic operations. In our case, not only it prevented massive bleeding during excision, but also allowed a fertility-sparing minimally invasive management.
\end{abstract}

\section{Keywords}

Pedunculated Fibroid, Prolapsed Leiomyoma, Uterine Artery Embolization, Hysteroscopy

\section{Introduction}

In literature, there are only a small number of reports for pedunculated submucosal fibroids [1] [2] [3] [4]. Submucosal fibroids account for approximately 15 - 
20 percent of total fibroids. The proportion of submucosal leiomyomas that prolapse through the cervix is uncertain. However an estimate of about $2 \%$ of submucosal leiomyomas is reported. In general, the size ranges approximately 1 to $6 \mathrm{~cm}$ in diameter. Larger fibroids are unlikely to prolapse through the cervix. The most common clinical presentation is severe vaginal bleeding [4].

Prolapsed leiomyomas are typically removed via vaginal myomectomy, although when significantly large, they pose a surgical challenge. Most cases in literature were treated with either scheduled abdominal total hysterectomy, or emergency abdominal hysterectomy due to uncontrollable bleeding after a vaginal approach. Interventional radiology, has been available in our department for years, however it is infrequently used by gynecologists. The preoperative uterine artery embolization (UAE) is a safe procedure that becomes more and more popular in complicated gynecologic surgery.

\section{Case Presentation}

We present a 38-year-old nulliparous woman who was referred to our department for robotic fibroid enucleation. In the past, this woman had been operated three times for fibroid enucleations. She had undergone an abdominal laparotomy ( 9 years earlier), a laparoscopy (4 years earlier) in another institution. Finally, she had undergone a successful hysteroscopy only 7 months earlier, for a submucous fibroid which was removed resectoscopically, followed by insertion of a progesterone-releasing (intrauterine device) IUD, to facilitate endometrial healing. Recently she experienced again intense and irregular bleeding (metrorrhagia) for which she performed ultrasonography and subsequently a magnetic resonance imaging (MRI). The MRI showed several intramural fibroids, the largest of which sized 12-cm, filling the lesser pelvis (Figure 1(a)). The follow-up MRI revealed ultimate changes (Figure 1(b)).

Interestingly, the patient's chief complaint was a recent intense malodorous vaginal discharge, irresponsive to oral antifungal therapy, rather than other common symptoms of the large fibroids. To our surprise, a speculum examination

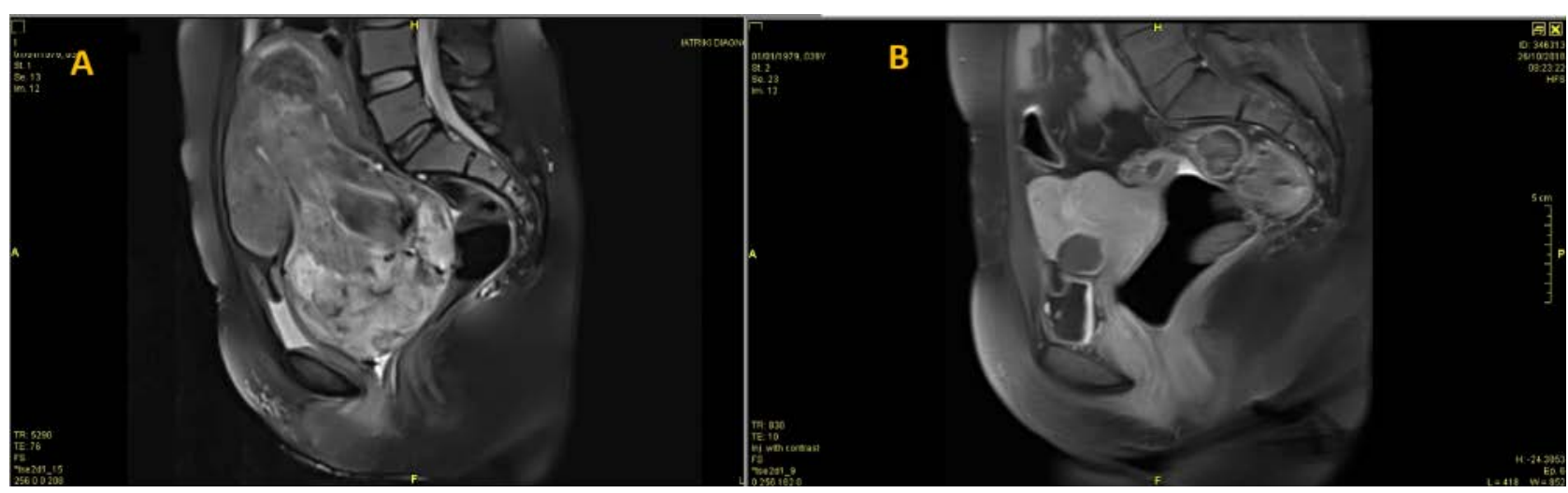

Figure 1. (a): MRI on presentation. Sagittal plane. The fundus extends as high as L3. The lesser pelvis is filled with a large fibroid with degenerative necrosis. The cervix is not visible. (b): MRI at 6-months follow up. Sagittal plane. The pelvic anatomy is restored. Only few small intramural fibroids are seen. The endometrial cavity is distinguished. 
was impossible, as a huge intravaginal mass, reaching the lower third of the vagina, overfilled the canal (Figure 2(a)). There was an intense foully smelling brownish vaginal discharge, containing clots and pieces of desquamated necrotic tissue. The surface of the mass was firm, irregular, friable and bloody. The cervix could not be seen, nor palpated with digital examination on either side around the mass. The examination was very uncomfortable for the patient. Naturally, the IUD could not be seen.

On admission, the patient was mildly febrile (37.5C) and anemic (Hct (hematocrit) $27.8 \%, \mathrm{Hb}$ (hemoglobin) $8.1 \mathrm{~g} / \mathrm{dL}, \mathrm{MCH}$ (mean corpuscular $\mathrm{Hb}$ ) $23.1 \mathrm{pg}$ ). Intravenous ceftriaxone and clindamycin were employed and rapid resolution (within 48 hours) with respect to the amount and odor of the vaginal secretions, parametrial sensitivity as well as the fever. The vaginal culture later revealed communal infection with Proteus mirabilis. Transvaginal ultrasonography (TVU) showed a highly vascular mass, this is why a punch biopsy was not attempted in the ambulatory ward. A cervical speculum cytologic examination was negative.

Patient also claimed, she experienced frequently excessive vaginal bleeding, sometimes with thick clots, therefore it could be possible that the IUD might have been expelled unnoticed. The IUD could not be visualized in the abdominal MRI, or the TVU. An abdominal $x$-ray identified the IUD within pelvis, dislocated ventrally and inferiorly, just behind the pubic symphysis (Figure 2(b)).

On the 4th day of hospitalization, the patient gave written informed consent for bilateral uterine artery embolization, with subsequent transvaginal approach

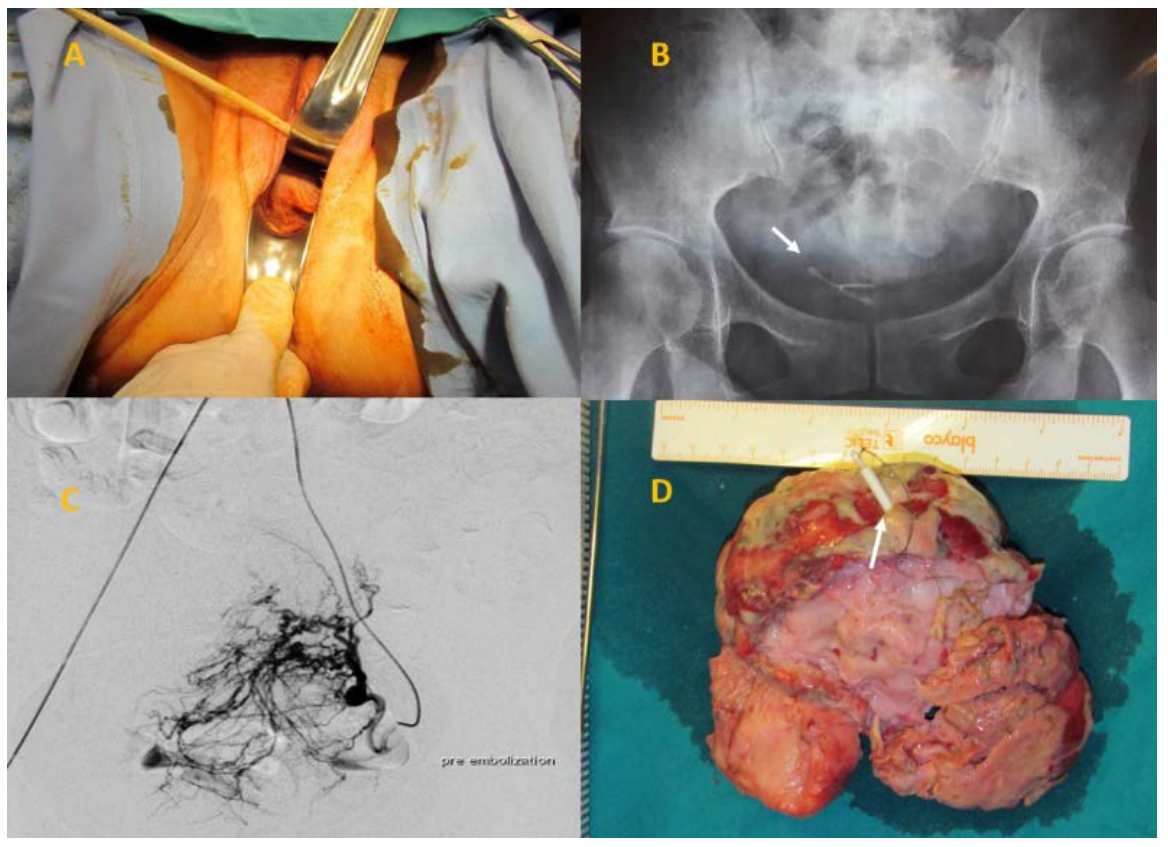

Figure 2. (a): Speculum examination under general anesthesia. The mass reaches the lower third of the vagina, (b): Pelvic x-ray. White arrow points at the IUD, (c): Angiography prior the embolization, showing a strongly right dominant vascular supply of the fibroid, (d): The specimen. 
to remove the mass. The aim of the embolization was, to minimize bleeding during surgery, which would take place 24 hours later. After standard preparation, arterial access was obtained by the retrograde puncture of her right femoral artery and insertion of a 5-Fr sheath (Radifocus Introducer II, Terumo Corporation, Tokyo, Japan). Angiography of the left internal iliac artery was performed in a crossover maneuver with a 4-Fr diagnostic catheter (Cobra II, Terumo Corporation). Angiography showed that the fibroid had a strongly right dominant vascular supply (Figure $2(\mathrm{c})$ ). Access to her right uterine artery was also obtained using a coaxial technique and a 5-Fr diagnostic catheter (Simmons II, Terumo Corporation). Bilateral embolisation of uterine arteries was subsequently performed, using calibrated microspheres measuring $500-700 \mu \mathrm{m}$ and 700 - $900 \mu \mathrm{m}$ (Embosheres ${ }^{\circledR}$, Merit Medical Systems ${ }^{\oplus}$, Utah, USA).

The following day, a diagnostic hysteroscopy was performed which visualized a fully dilated cervix, an exposed endometrium and the IUD along the cranial aspect of the mass. Subsequently, the thick peduncle of the fibroid was carefully transected with rigid resectoscope (KARL STORZ SE \& Co, Germany). Hysteroscopy was totally bloodless, with a clear visual field during resection. Following transection of the broad stalk, the fibroid was readily delivered through the vagina. Upon removal of the mass, the entire uterine cavity was wide open, and evident through a fully dilated cervix, with direct speculum examination.

Following the dissection of the base, the fibroid was "delivered" from the vagina intact. The IUD was identified on the surface of the mass, partly embedded within the fibroid (Figure 2(d)). The procedure was bloodless, and no transfusion took placeintra- or post-operatively. The post-operative period was uneventful and patient was discharged 48 hours later. In total, the patient was hospitalized for 7 days.

At 6-months follow up, the patient had fully recovered and was gratified. The Hct had risen to 41 (without transfusion), menses became regular, although bleeding now lasted for only two days. Ultrasonography showed a normal functioning endometrium $7 \mathrm{~mm}$ in thickness. The follow-up MRI revealed ultimate changes (Figure 1(b)). The pelvic anatomy was restored.

\section{Discussion}

The authors have received a scientific committee approval and patient consent for publishing this case report.

On admission, it was difficult to define the origin of the intravaginal mass. The MRI examination could not distinguish whether the fibroid at the bottom of the uterus lied intraperitoneally within the lesser pelvis, or intravaginally. The cervix could not be visualized. The patient's history was a strong argument, that this could not have been a newly developed submucosal fibroid. It seemed more logical, that an already existing intramural fibroid, lying within the uterine corpus, found access into the endometrial cavity, due to the shedding and thinning of the endometrium caused by the resectoscopic myomectomy. Subsequently, 
uterine contractions, caused gradual cervical dilatation and effacement, until finally the fibroid was expelled into the vagina, held in place by a thick stalk. A similar mechanism has been proposed in literature, during puerperium [5]. Another suggested mechanism is by direct perforation of the IUD, into the intramural leiomyoma or within the fibroid capsule facilitating the breakdown and entry of the intramural myoma into the cavity. Although in literature there are several reports for misplaced IUDs, or perforations of the myometrium, to our knowledge, there is no evidence for direct IUD insertion within a rigid fibroid.

Prolapsed leiomyomas are typically removed via vaginal myomectomy. Complications of vaginal myomectomy for a prolapsed fibroid are rare, based upon the few available data. In two retrospective series, there were no complications. The most likely potential complication of vaginal myomectomy is excessive bleeding from the fibroid site [1] [3]. Very large prolapsed fibroids are usually accompanied by uterine prolapse. A vaginal hysterectomy is usually the method of choice for these cases [6].

There are only a couple of reports for huge prolapsed intravaginal leiomyomas, without uterine prolapse. In one of these, a giant prolapsed leiomyoma, sized $12 \mathrm{~cm}$, in a 45-year-old woman, was treated with hysterectomy, due to excessive bleeding after vaginal myomectomy. The authors recommended clamping the fibroid's stalk, if possible, before attempting to remove it [7]. In another report, a similar case with an intravaginal fibroid of $11 \times 9 \times 7 \mathrm{~cm}$, in a 39-year-old woman (G2, P2), a total abdominal hysterectomy was performed to avoid bleeding [8]. In our case, the uterus did not prolapse. Somehow, it was anchored within pelvis, due to the increased size of the corpus uteri, caused by the presence of several smaller intramural fibroids.

To our knowledge, there is no report for uterine-preserving approach. A minimal invasive surgery would be challenging if possible at all, because the fibroid was below the cervical plane, therefore an intra-abdominal approach would require a full thickness dissection of the uterus, to access and transect endometrium. A transvaginal approach, seemed more promising, however, significant bleeding was anticipated, if which is uncontrollable, it would prompt urgent hysterectomy. After careful consideration, it was decided to perform bilateral uterine artery embolization followed by surgical excision. In our institution, we have experienced department of invasive radiology, in collaboration with which, we have already performed a series of uterine artery embolizations for leiomyomas. Indeed, all previous cases involved intramural fibroids.

Uterine artery embolization (UAE) is a well-established uterine preserving and minimally invasive therapy for symptomatic fibroids. There is strong evidence for safety and efficacy, with low rates of complications [9] [10]. Although in the past, UAE was avoided in large leiomyomas, recent studies have demonstrated its safety for masses over $10 \mathrm{~cm}$ [9]. In general, UAE is avoided for pedunculated fibroids, since it is associated with higher rates of stalk necrosis and inflammation requiring surgical intervention [9]. With respect to fertility, fol- 
lowing UAE, little is yet known, due to a small amount of available data. In one randomized trial, the authors demonstrated that myomectomy resulted in better reproductive outcomes compared with UAE [11]. In another study, women without infertility factors showed an encouraging delivery rate after UAE. Furthermore, UAE may not impair fertility when compared with abdominal myomectomy, however, data is insufficient to recommend UAE in nulliparous [12]. In our case, the aim of UAE was the control of bleeding during surgery. The patient refused a desire for fertility and gave written consent for possible future complications on fertility.

\section{Conclusion}

Uterine artery embolization (UAE) is a safe, well-established uterine preserving therapy for symptomatic, even large leiomyomas. Furthermore, pre-operative UAE, may be valuable in challenging gynecologic procedures, especially when heavy bleeding is anticipated.

\section{Conflicts of Interest}

The authors declare no conflicts of interest regarding the publication of this paper.

\section{References}

[1] Golan, A., Zachalka, N., Lurie, S., Sagiv, R. and Glezerman, M. (2005) Vaginal Removal of Prolapsed Pedunculated Submucous Myoma: A Short, Simple, and Definitive Procedure with Minimal Morbidity. Archives of Gynecology and Obstetrics, 271, 11-13. https://doi.org/10.1007/s00404-003-0590-x

[2] Faivre, E., Surroca, M.M., Deffieux, X., Pages, F., Gervaise, A. and Fernandez, H. (2010) Vaginal Myomectomy: Literature Review. Journal of Minimally Invasive Gynecology, 17, 154-160. https://doi.org/10.1016/j.jmig.2009.12.007

[3] Dicker, D., Feldberg, D., Dekel, A., Yeshaya, A., Samuel, N. and Goldman, J.A. (1986) The Management of Prolapsed Submucous Fibroids. Australian and New Zealand Journal of Obstetrics and Gynaecology, 26, 308-311. https://doi.org/10.1111/j.1479-828X.1986.tb01595.x

[4] Ben-Baruch, G., Schiff, E., Menashe, Y. and Menczer, J. (1988) Immediate and Late Outcome of Vaginal Myomectomy for Prolapsed Pedunculated Submucous Myoma. Obstetrics \& Gynecology, 72, 858-861. https://doi.org/10.1097/00006250-198812000-00009

[5] Nkwabong, E. (2018) An Intramural Uterine Fibroid Became Submucosal in the Puerperium-Proposed Probable Mechanism: A Case Report. Journal of Medical Case Reports, 12, 88. https://doi.org/10.1186/s13256-018-1624-0

[6] Newton, B.W. and Harmanli, O. (2016) Perplexing Presentation of Uterine Prolapse and a Prolapsed Pedunculated Leiomyoma. American Journal of Obstetrics \& Gynecology, 215, 799.e1-799.e2. https://doi.org/10.1016/j.ajog.2016.08.028

[7] Brito, L.G., Magnani, P.S., de Azevedo Trapp, A.E. and Sabino-de-Freitas, M.M. (2011) Giant Prolapsed Submucous Leiomyoma: A Surgical Challenge for Gynecologists. Clinical and Experimental Obstetrics and Gynecology, 38, 299-300.

[8] da Silva, B.B., da Silva-Sampaio, J.P. and Lopes-Costa, P.V. (2018) Huge Prolapsed 
Pedunculated Necrotizing Submucosal Leiomyoma. Journal of Minimally Invasive Gynecology, 25, 1128-1129. https://doi.org/10.1016/j.jmig.2018.01.026

[9] Kohi, M.P. and Spies, J.B. (2018) Updates on Uterine Artery Embolization. Seminars in Interventional Radiology, 35, 48-55.

https://doi.org/10.1055/s-0038-1636521

[10] Keung, J.J., Spies, J.B. and Caridi, T.M. (2018) Uterine Artery Embolization: A Review of Current Concepts. Best Practice \& Research Clinical Obstetrics \& Gynaecology, 46, 66-73. https://doi.org/10.1016/j.bpobgyn.2017.09.003

[11] Mara, M., Maskova, J., Fucikova, Z., Kuzel, D., Belsan, T. and Sosna, O. (2008) Midterm Clinical and First Reproductive Results of a Randomized Controlled Trial Comparing Uterine Fibroid Embolization and Myomectomy. CardioVascular and Interventional Radiology, 31, 73-8557. https://doi.org/10.1007/s00270-007-9195-2

[12] Torre, A., Fauconnier, A., Kahn, V., Limot, O., Bussierres, L. and Pelage, J.P. (2017) Fertility after Uterine Artery Embolization for Symptomatic Multiple Fibroids with No Other Fertility Factors. European Radiology, 27, 2850-2859.

https://doi.org/10.1007/s00330-016-4681-z 